Cinzia Cortesi · Pietro E. G. Foglia

Alberto Bettinelli • Mario G. Bianchetti

\title{
Prevention of cardiac arrhythmias in pediatric patients with normotensive-hypokalemic tubulopathy
}

\section{Current attitude among European pediatricians}

Received: 3 April 2003 / Accepted: 15 May 2003 / Published online: 12 June 2003

(C) IPNA 2003

\begin{abstract}
Potassium deficiency predisposes to cardiac arrhythmias culminating in syncope or sudden death. Because of the uncertainty related to the possible occurrence of such cardiac arrhythmias in the context of normotensive-hypokalemic tubulopathies, 19 European pediatric nephrologists with a large experience of normotensive-hypokalemic tubulopathies were asked to answer a questionnaire. The responses suggest that inherited normotensive-hypokalemic tubulopathies per se do not strongly predispose to dangerous cardiac arrhythmias. However, cardiac arrhythmias may be acutely precipitated by drugs that prolong the QT interval, by diarrhea, or vomiting, and perhaps even by physical activity. Finally, the likelihood of dangerous arrhythmias in normotensive-hypokalemic tubulopathy is currently unknown.
\end{abstract}

Keywords Bartter disease $\cdot$ Hypokalemia . Hypomagnesemia $\cdot$ Sudden cardiac death

C. Cortesi · P. E. G. Foglia · M. G. Bianchetti (

Division of Nephrology,

University Children's Hospital,

Inselspital, 3010 Berne, Switzerland

e-mail: mario.bianchetti@insel.ch

Tel.: +31-632-9512, Fax: +31-632-9444

C. Cortesi · P. E. G. Foglia · A. Bettinelli · M. G. Bianchetti Pediatric Renal Unit,

University of Milan Medical School,

Clinica De Marchi, Milan, Italy

\section{A. Bettinelli}

Division of Pediatrics,

Leopoldo Mandic Hospital,

Merate, Italy

M. G. Bianchetti

Division of Pediatrics,

San Giovanni Hospital,

Bellinzona, Switzerland
The term normotensive-hypokalemic tubulopathy (or the eponym Bartter syndrome) is used to describe inherited renal disorders with a characteristic set of metabolic abnormalities that include hypokalemia and alkalosis. Many genetically distinct normotensive-hypokalemic tubulopathies (with or without concomitant hypomagnesemia) have been described, and recent studies have identified the underlying loss-of-function mutations in various genes [1, 2]. Potassium and magnesium deficiencies increase cardiac excitability and predispose to cardiac arrhythmias, culminating in syncope or sudden death $[3,4,5,6]$. Hypokalemia, with or without concomitant hypomagnesemia, is sometimes severe in normotensive-hypokalemic tubulopathies. Nonetheless, little is known about the occurrence of malignant arrhythmias in these conditions, with the exception of a recently reported boy with an unclassifiable normotensivehypokalemic tubulopathy who suddenly died [7]. In addition, the electrocardiographic QT interval, a marker of cardiac excitability, is often prolonged in this condition $[8,9]$.

Because of the uncertainty related to the possible occurrence of cardiac arrhythmias culminating in syncope or sudden death in the context of normotensivehypokalemic tubulopathies, we asked a group of pediatric nephrologists with a large experience of normotensivehypokalemic tubulopathies currently active in European countries to answer eight questions related to the prevention of dangerous cardiac arrhythmias in this condition. Nineteen nephrologists answered the questionnaire. ${ }^{1}$

The first question focused on the use of a standard electrocardiogram and measurement of the QT interval (at least once) in children with normotensive-hypokalemic tubulopathy. Five nephrologists never use a standard electrocardiogram, 10 sometimes do, and 4 very often or even always do.

The second question focused on the use of Holter electrocardiography for $24 \mathrm{~h}$ in children with normotensive-hypokalemic tubulopathy. Most nephrologists $(n=14)$ never use this, while a minority $(n=4)$ sometimes use Holter electrocardiography in this condition.

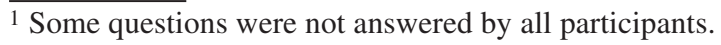


The third question focused on the possible supplementation with potassium or magnesium salts and treatment with cyclo-oxygenase inhibitors or potassiumsparing diuretics based on the electrocardiographic (or Holter) features. The answer to this question was very clear: 16 nephrologists stated that electrocardiographic features never influenced their therapeutic attitude.

Several drugs prolong the QT interval and have been associated with cardiac arrhythmias [10]. In addition to some anti-arrhythmic agents, QT-prolonging drugs include, among others, some antihistamines, some macrolides, some antifungals, some psychotropics, $\beta_{2}$-agonists, and cisapride. Sine the potential of these drugs to induce arrhythmias is favored by the imbalance in potassium or magnesium levels, a case might well be made for avoiding the use of these drugs in normotensive-hypokalemic tubulopathies. The 19 pediatric nephrologists considered (answer to question 4) that avoiding these drugs in these tubulopathies is rather important $(n=1)$, important $(n=5)$, or very important $(n=7)$. In contrast, a large subset does not have a corresponding clear-cut opinion $(n=6)$.

Hypokalemia and, to a lesser extent, hypomagnesemia often occur in children with acute diarrhea or vomiting. Consequently, it is tempting to assume that in normotensive-hypokalemic tubulopathies the risk of severe cardiac arrhythmias is particularly high in the presence of diarrhea or vomiting. Of the 19 pediatric nephrologists involved, 4 consider that diarrhea or vomiting slightly increases the tendency towards cardiac arrhythmias in normotensive-hypokalemic tubulopathies, 9 that acute diarrhea or vomiting strongly increase the tendency towards cardiac arrhythmias, and 5 that acute diarrhea or vomiting very strongly increase the tendency towards cardiac arrhythmias (question 5).

In patients with congenital QT prolongation, dangerous cardiac arrhythmias often occur, mostly precipitated by physical activity [10]. As a consequence, patients with congenital causes of QT prolongation are currently excluded from competitive sports. A case could be made for excluding patients with normotensive-hypokalemic tubulopathies from competitive sports (question 6). A large subset $(n=6)$ of the pediatric nephrologists had no clear-cut opinion. Three of the participants stated that excluding patients with normotensive-hypokalemic tubulopathies from competitive sports is unimportant. The opinions in the remaining 10 nephrologists were divided: 4 stated that excluding patients from competitive sports is rather important, 4 that it is important, and 2 that it is very important.

Of the 19 nephrologists, 17 feel (answer to question 7) that further investigations are important or even very important to assess the true hazard of dangerous arrhythmias in patients with normotensive-hypokalemic tubulopathies. Finally, at least 14 recommend (question 8) setting up a working group of experienced cardiologists and nephrologists to develop practical guidelines for the cardiac work-up and management of children and adolescents with normotensive-hypokalemic tubulopathy.

In conclusion, pediatric nephrologists are of the opinion that inherited normotensive-hypokalemic tubulo- pathies per se do not strongly predispose to dangerous cardiac arrhythmias. However, in normotensive-hypokalemic tubulopathies dangerous cardiac arrhythmias may be acutely precipitated by medical treatment with drugs that prolong the QT interval, by diarrhea or vomiting, two further causes of hypokalemia in childhood, and perhaps even by physical activity (competitive sports). The attitude of pediatric nephrologists towards the risks of cardiac arrhythmias in children with normotensive-hypokalemic tubulopathy likely reflects the assumption that cardiomyocytes adapt to chronic hypokalemia but do not adapt to acute hypokalemia. Although cardiac excitability has been related to extracellular electrolyte imbalance, it is the altered gradient across the cell membrane that modulates cardiac excitability. In acute hypokalemia, intracellular potassium levels are relatively stable and altered potassium gradients are largely dependent on extracellular potassium levels. In chronic hypokalemia, however, intracellular potassium levels are reduced, suggesting that the potassium gradient across the cardiomyocyte membrane is not dependent only on extracellular potassium levels $[5,8,9]$. Finally, the answers reflect the fact that the likelihood of dangerous arrhythmias in normotensive-hypokalemic tubulopathy is currently unknown.

Acknowledgements We acknowledge the support of the Associazione Bambino Nefropatico. The questionnaire was answered by the following: P. Bertolani (Modena, Italy), F. Cachat (Lausanne, Switzerland), D.A. Caringella (Bari, Italy), G. Colussi (Varese, Italy), G. Deschênes (Paris, France), F. Emma (Rome, Italy), M. Fischbach (Strasbourg, France), C. Holmberg (Helsinki, Finland), E. Levtchenko (Nijmegen, The Netherlands), C. Loirat (Paris, France), T.J. Neuhaus (Zürich, Switzerland), P. Niaudet (Paris, France), W. Proesmans (Leuven, Belgium), J. Rodríguez-Soriano (Bilbao, Spain), J. Strehlau (Hannover, Germany), B. Tönshoff (Heidelberg, Germany), and A.M. Wingen (Essen, Germany).

\section{References}

1. Bettinelli A, Vezzoli G, Colussi G, Bianchetti MG, Sereni F, Casari G (1998) Genotype-phenotype correlations in normotensive patients with primary renal tubular hypokalemic metabolic alkalosis. J Nephrol 11:61-69

2. Zelikovic I (2001) Molecular pathophysiology of tubular transport disorders. Pediatr Nephrol 16:919-935

3. Fiset C, Kargacin ME, Kondo CS, Lester WM, Duff HJ (1996) Hypomagnesemia: characterization of a model of sudden cardiac death. J Am Coll Cardiol 27:1771-1776

4. Materson BJ (1997) Diuretics, potassium, and ventricular ectopy. Am J Hypertens 10:68S-72S

5. Weiner ID, Wingo CS (1997) Hypokalemia-consequences, causes, and correction. J Am Soc Nephrol 8:1179-1188

6. Vannacci A, Baronti R, Masini E, Ravaldi C, Ricca V (2002) Anorexia nervosa and the risk of sudden death. Am J Med $112: 327-328$

7. Zanolari Calderari M, Vigier RO, Bettinelli A, Bianchetti MG (2002) Electrocardiographic QT prolongation and sudden death in renal hypokalemic alkalosis. Nephron 91:762-763

8. Blomstrom-Lundqvist C, Caidahl K, Olsson SB, Rudin A (1989) Electrocardiographic findings and frequency of arrhythmias in Bartter's syndrome. Br Heart J 61:274-279

9. Bettinelli A, Tosetto C, Colussi G, Tommasini G, Edefonti A, Bianchetti MG (2002) Electrocardiogram with prolonged QT interval in Gitelman disease. Kidney Int 62:580-584

10. Li H, Fuentes-Garcia J, Towbin JA (2000) Current concepts in long QT syndrome. Pediatr Cardiol 21:542-550 